\title{
Focal-plane wavefront sensing for segmented telescope
}

\author{
E. Delavaquerie ${ }^{1,3, a}$, F. Cassaing ${ }^{1,3}$, and J.-P. Amans ${ }^{2,3}$ \\ 1 ONERA-DOTA, Unité HRA, Châtillon, France \\ 2 GEPI, Observatoire de Paris, CNRS, Université de Paris Diderot, Meudon, France \\ 3 Groupement d'intérêt scientifique PHASE between ONERA, Observatoire de Paris, CNRS and Uni- \\ versité Diderot
}

\begin{abstract}
To achieve the ultimate resolution of future ELTs, the segments of their primary mirror must be aligned. This paper present focal plane (FP) wavefront sensing techniques that allow to perform piston measurements between the segments. Those techniques have the advantage of using images that are the result of the interference of all the segments. This makes FP measurements capable of handling complex apertures. New developments at ONERA allow us to apply FP measurements for different applications. We present an upgrade of the usual Shack-Hartmann pairwise technique. We also show that phase diversity is able to cophase an ELT. Eventually, we show our current developments in term of analytical algorithms and our new bench, NIRTA, which is under integration at ONERA.
\end{abstract}

\section{Introduction}

Future ELTs will have their primary mirror segmented. If not perfectly aligned, the segments will seriously affect the point spread function of the telescope and lead to blurred images at their focalplane. Those misalignments, mostly piston, tip and tilt, must be measured in order to align precisely the primary mirror's surface. The figure 1 shows an example of an ELT-like telescope with a $\lambda / 4 \mathrm{rms}$ error consisting in piston aberration on its segments. Obviously, the affected image is not usable and needs to be corrected.
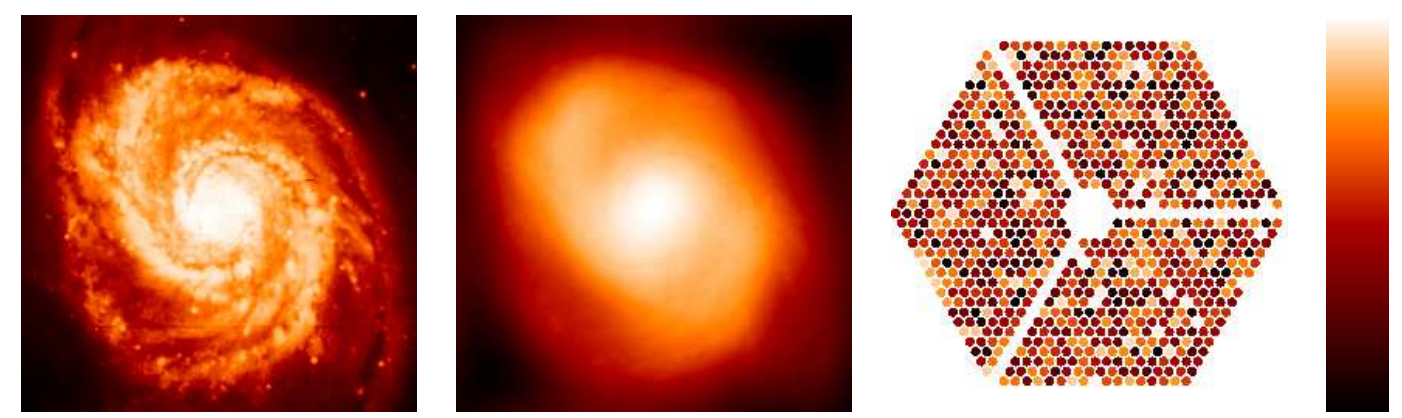

Fig. 1. Effect of a bad cophasing on the focal-plane image for an ELT-like segmented pupil. Left: focal-plane image with a perfect cophasing. Middle: same data but with a $\lambda / 4 \mathrm{rms}$ error on the phasing of the segments. Right: considered pupil.

In consequence, a cophasing of the segmented mirror is needed, i.e. the misalignments must be measured by a dedicated device and later be compensated. Indeed usual Adaptive Optics (AO) systems are not suitable to detect directly piston aberrations, which is the most challenging task when cophasing segmented pupils. The Active Phasing Experiment [1-3] managed to compare four WFS

\footnotetext{
a e-mail: enguerran.delavaquerie@onera.fr
} the original work is properly cited. 
for the European-ELT: a pyramid sensor, a curvature sensor, a phase filtering sensor and a ShackHartmann (SH) sensor. Each of them are modified versions of the usual sensors. In a same approach, it is planed to use a Shack-Hartmann WFS for the cophasing of the primary mirror of the Thirty Meter Telescope [4]. This shows that pupil-Plane (PP) Wavefront Sensors (WFS) are the most considered devices to cophase segmented mirrors. On the other side, the James Web Space Telescope (JWST) plans to use a Focal-Plane (FP) WFS for the fine cophasing of its segments [5]. The advantage of using a focal-plane wavefront sensor is that the recorded images are the results of the interference of all the segments. As a consequence, and in opposition to pupil plane techniques, a FP-WFS can handle missing segments, the presence of a central obscuration, or a spider. Furthermore the method requires little or no optics other than the imaging sensor, even for a lot of segments, and is not subject to differential path errors.

This paper shows the developments we are leading at ONERA concerning focal plane wavefront sensors applicated to the case of ELTs. In a first part, we will see a new algorithm that allows to improve the pairwise measurement of the classical SH-WFS used for segmented telescopes. We will show that another algorithm, phase diversity, can handle a larger number of segments. Eventually, we will see the current developments at ONERA in term of analytical algorithms and future experiments.

\section{Analytical piston estimation with a Shack-Hartmann WFS}

The Shack-Hartmann WFS is usually seen as a pupil-plane WFS which measures the local slopes of an incoming wavefront. Those slopes are measured using center of gravity (COG) algorithms and a geometrical data model for the image formation. However, this device is unable to measure piston steps because of the discontinuity of the incoming wavefront. Indeed, the only way to produce a signal which is function of a piston step between two segments, is to let a lens of the SH mask straddling the segments. In figure 2-left, we can see a possible implementation of such a mask: in a pupil plane we put a lenslet array which consists in round lenslets located at the edges of the projected segments. At their focal-plane a signal is produced, function of the incoming piston step. This diffraction pattern is shown for four different piston steps from 0 to $\pi$ rad in figure 2-right. When the two segments are in phase, the diffraction figure is the usual Airy pattern and when a piston step exists, the pattern is heavily affected, but not its COG. In the existing algorithms, this signal is cross-correlated with calibrated pre-computed patterns in order to retrieve the phase difference [4,6-8].
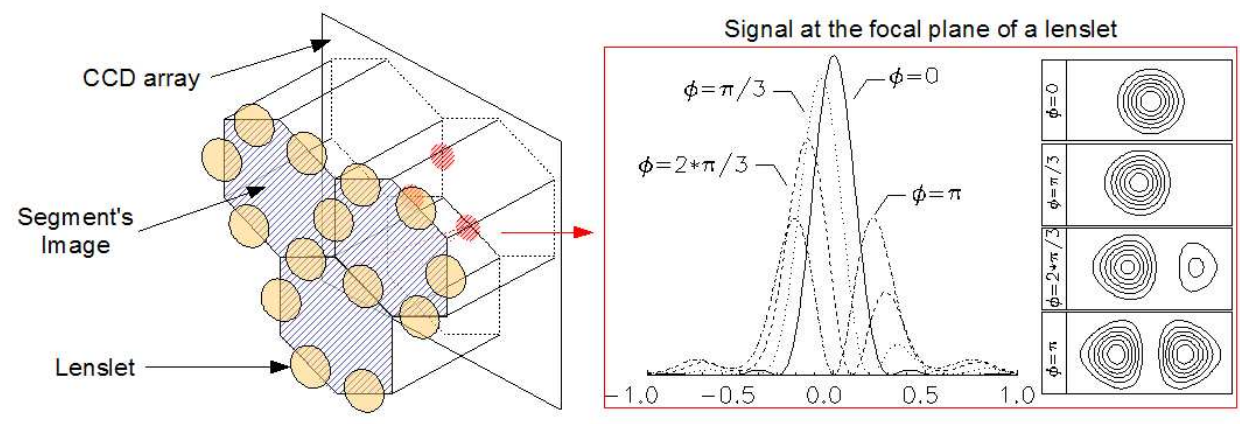

Fig. 2. Left: pupil configuration. The segments are imaged onto a lenslet array. Each lenslet allows to perform piston measurements between the straddled segments. Right: Point Spread Function shown for four piston steps as a function of the longitudinal dimension in the focal-plane of the Shack-Hartmann mask.

This method has proved its efficiency, but a more efficient way exist to exploit the diffraction pattern. We have developed a new single-step analytical algorithm which, from a single focal-plane image, retrieves the piston step information between two segments. 

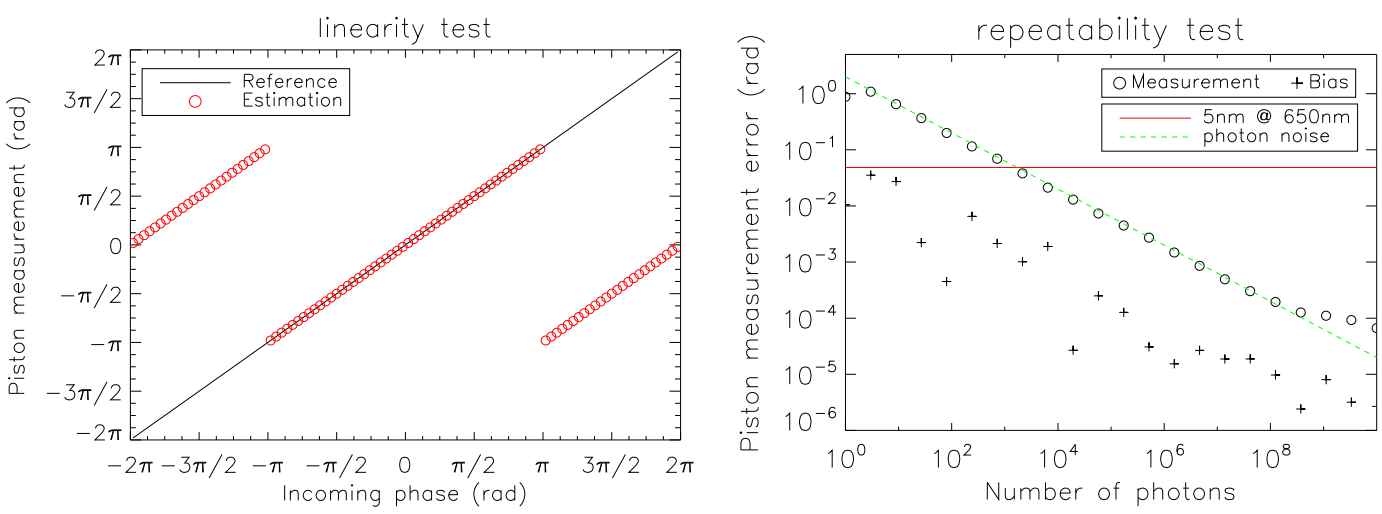

Fig. 3. Left: piston measurements for an increasing piston difference between two segments. Right: RMS measurement precision as a function of the incoming number of photons and estimated bias.

This analytical algorithm will be described in [9]. To have an idea of its performance, we have performed a linearity test at high flux $\left(10^{6}\right.$ photons/frame for a $16 \times 16$ matrix $)$ with an incoming phase ramp included in the $\pm 2 \pi$ range (see figure 3-left). Except for a phase wrapping around $\pm \pi$, which is inherent to piston measurements, the measurements (circles) fit fairly well the ramp (solid line) with a mean bias of $1.410^{-4}$ rad. The capture range can be extended with, for example, 2 wavelengths techniques [10] to reach several microns.

Classical phase estimation algorithms have their accuracy which is function of the incoming number of photons. We have plotted on figure 3 the rms estimation error $\sigma$ (circle) in closed loop mode $(\phi=0)$ and the corresponding bias $\Delta$ (cross) for different flux, with:

$$
\sigma_{\phi=0}=\sqrt{\overline{(\hat{\phi}-\overline{\hat{\phi}})^{2}}}, \text { and } \Delta=\overline{\hat{\phi}}-\phi_{\text {true }}
$$

$\hat{\phi}$ denotes an estimated wavefront and the operator ${ }^{-}$an averaging over the noise samples. The horizontal line corresponds to an error of $\lambda / 130$ (5 nm @ $\lambda=650 \mathrm{~nm})$, a typical error budget. Each point is the average of 500 noise realizations, and we assume a perfect detector (no readout noise). The algorithm follows the photon noise regime in $\sigma \simeq \alpha / \sqrt{N}$ with $\alpha \simeq 1.7$ (dashed line) versus $\alpha \simeq \sqrt{2}$ for typical piston measurements. The algorithm is also barely affected by the bias. We can also point out that the RMS error corresponding to $\lambda / 130$ is reachable for a flux of the order of $10^{3}$ photo-electrons per frames.

This single measurement by itself is not able to measure the phase of a segmented mirror. But, by using all the individual measurements permitted by the lenslet array, the full piston map of a segmented aperture can be reconstructed. Our algorithm can be easily implemented in existing device and permits a direct exploitation of the diffracted signal at the focal plane of the lenslets of the Shack-Hartmann.

\section{Phase diversity as a cophasing sensor for ELTs.}

Section 2 has shown results from a technique using only one image to retrieve piston information between two segments. In the case of an ELT-like aperture such as the one drawn on figure 1-right, this technique is no longer usable because of possible sign ambiguities. However, by using a second image with an additional known aberration such as defocus, it is possible to estimate without ambiguity the incoming phase, even with an extended source. This technique, called Phase Diversity (PD) is described on figure 4.

The goal of PD is to find the aberrated phase $\phi_{a}$ from the two recorded images. The data model is given by:

$$
\begin{aligned}
& i_{1}=\left(o * h_{1}\right)+n_{1} \text { with } h_{1}=\left|F T^{-1}\left\{P . e^{j \phi_{a}}\right\}\right|^{2}, \\
& i_{2}=\left(o * h_{2}\right)+n_{2} \text { with } h_{2}=\left|F T^{-1}\left\{P . e^{j\left(\phi_{a}+\phi_{d}\right)}\right\}\right|^{2} .
\end{aligned}
$$




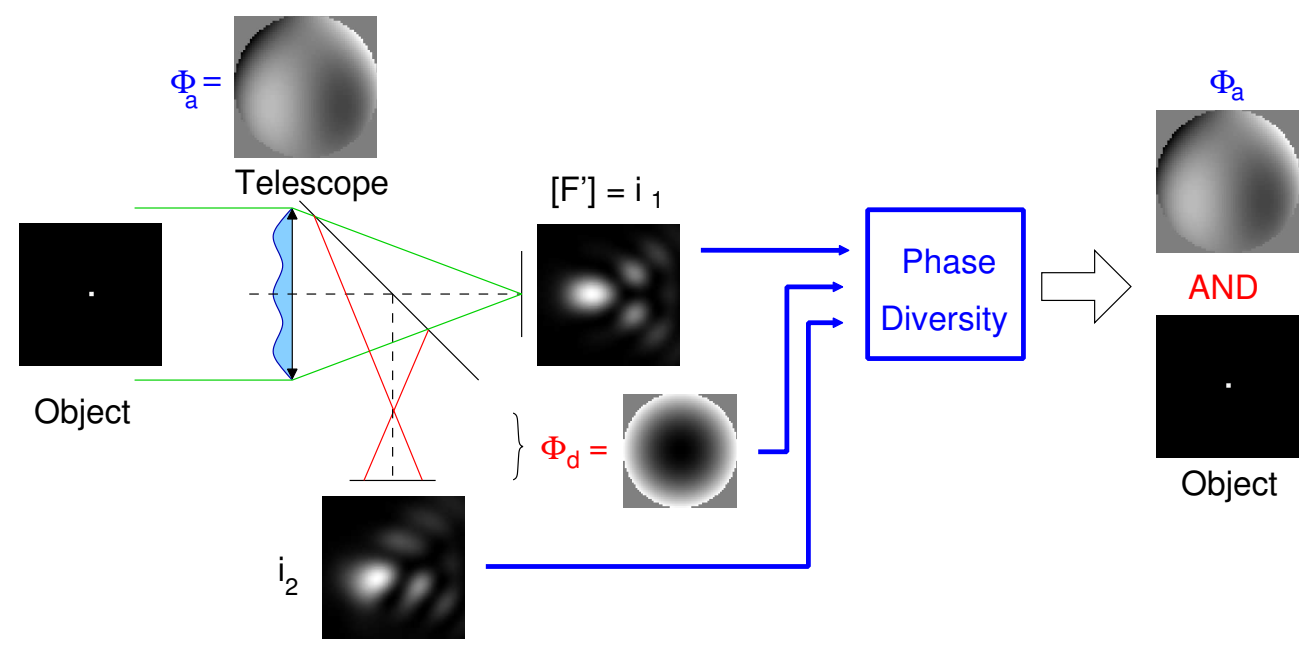

Fig. 4. Phase Diversity Scheme. The telescope has an aberration $\phi_{a}$ which modify the focal plane image $i_{1}$ (in our case, the image should be an airy disk). Another image $i_{2}$ with aberration $\phi_{d}$ allows to perform phase diversity measurements. By this way, the aberrations of the algorithm can be retrieve together with the initial object.

The images $\left(i_{1}\right.$ and $\left.i_{2}\right)$ are the convolution $(*)$ of the source intensity distribution $o$ and the point spread functions $\left(h_{1}\right.$ and $h_{2}$ ), plus noises $\left(n_{1}\right.$ and $n_{2}$, photon or detector noise). $F T^{-1}$ is the inverse Fourier transform operator. $P$ is the pupil function and $\phi_{d}$ the introduced diversity. The estimation of $\phi_{a}$ can be solved by the minimization of a given criterion, for example maximum likelihood estimator or maximum a posteriori estimator [11].

As already seen on section 1, the JWST will use phase diversity to cophase its 18 segments. However, it is not obvious that PD can cophase an ELT with its hundreds of segments. We performed a simulation on an ELT configuration (fig. 1-right) to evaluate the measurements accuracy as a function of the number of photons (fig. 5). The pupil is sampled in a $256 \times 256$ pixel matrix and each point is the average of 20 photon noise samples.

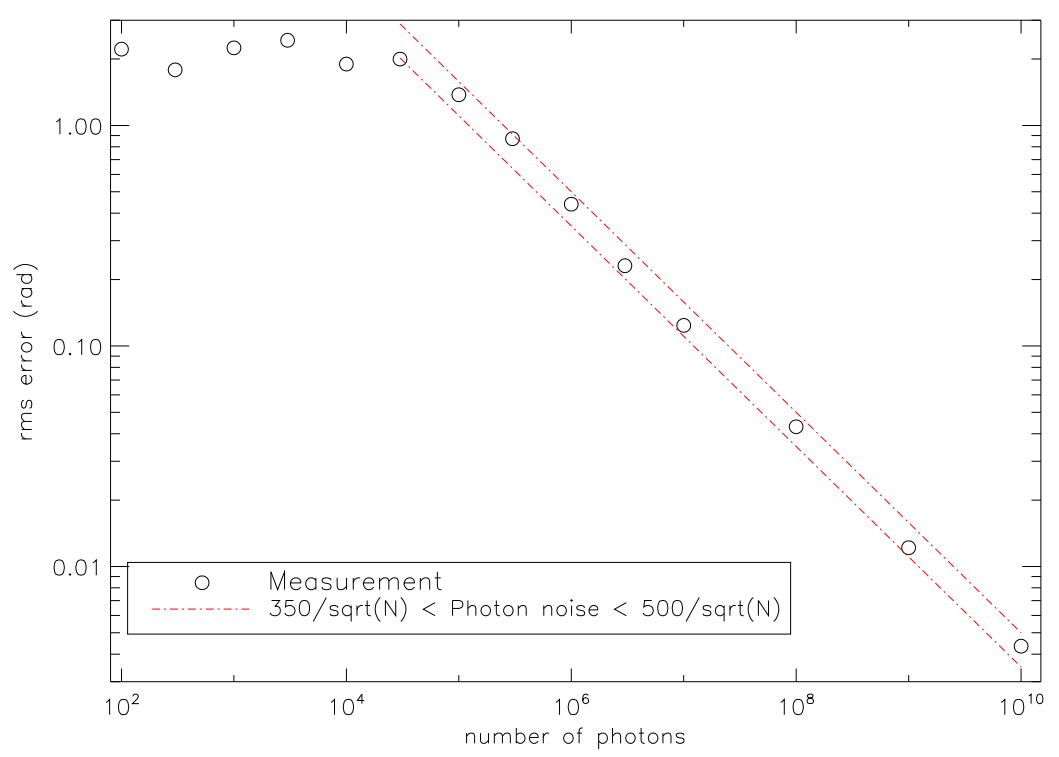

Fig. 5. RMS measurement precision as a function of the incoming number of photons. In the photon noise regime, the error scales in $\alpha / \sqrt{N_{\text {phot }}}$, with $\alpha$ between 350 and 500. 
As described on section 2, we can clearly see the photon noise regime. We consider a telescope of global transmission $\tau \simeq 0.2$ and a diameter of $42 \mathrm{~m}$. If the source is a $G 0$ star of magnitude 12 with a spectral bandwidth of $40 \mathrm{~nm}$, we found that the residual cophasing error is equal to $4 \mathrm{~nm} @ \lambda=$ $650 \mathrm{~nm}\left(10^{8} \mathrm{phot} / \mathrm{frame}\right)$ and $20 \mathrm{~nm} @ \lambda=1300 \mathrm{~nm}\left(10^{7} \mathrm{phot} /\right.$ frame $)$. Those results involve long exposure images and a pre-compensation by adaptive optics. In a previous work [12] we have proved that with an AO residual error of 1 radian rms it is worth to use PD to cophase the instrument.

Even with a large number of segments, phase diversity is able to perform its measurements. It is a powerful tool that is easy to implement (it only needs a good camera and a "diversity generator"). However, to perform the simulations presented above, we used iterative algorithm that needs a great amount of computations, therefore, time. In order to use PD in real-time applications, we need to develop faster algorithms.

\section{Current developments}

So, there is a big interest in fast algorithm that use FP images. We have already developed analytical FP algorithms which need a small phase assumption $[13,14]$. Now we are taking a new approach that will overcome this last problem. Our new algorithms will allow real-time measurements of the pistons aberrations of a segmented aperture, but also real-time image restoration.

In parallel of those developments, we are building a bench called NIRTA that will include a mirror with 19 segments movable on piston, tip and tilt (see figure 6). This mirror, which is designed by GEPI, is an upgrade of our previous 3-segment mirror of the BRISE bench [15]. NIRTA will also comprise an OLED emitting device that will act as an extended source. The bench will allow us to test our analytical algorithms and to test new concepts of telescopes array.
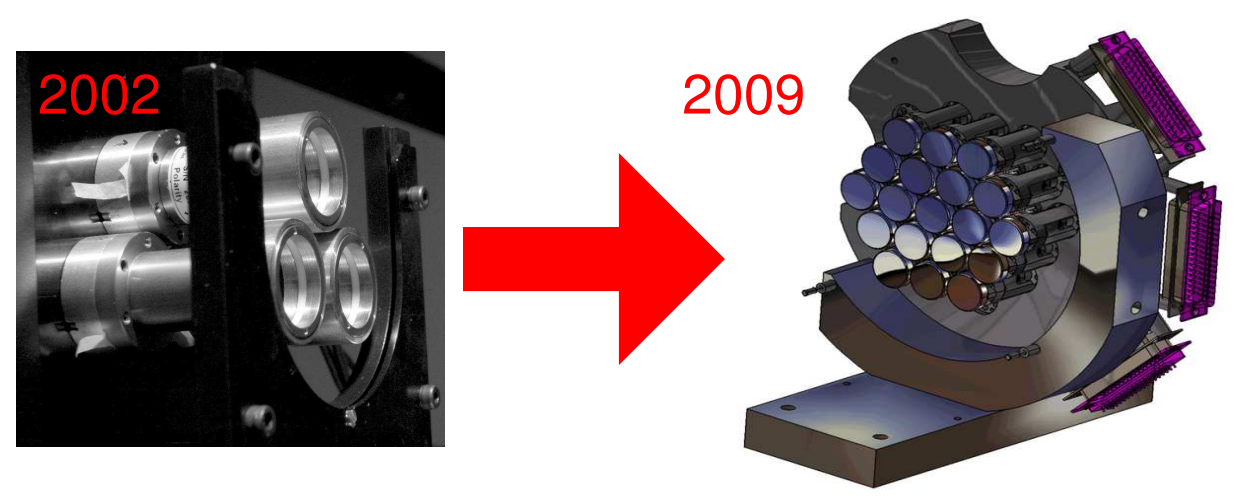

Fig. 6. Left: 3-segment mirror of the BRISE bench. 2 segments can be moved in piston tip and tilt, the third one is for reference. Right: 9-segments mirror of the NIRTA bench. All but one segments can be moved in piston, tip and tilt.

\section{Conclusions}

Cophasing segmented telescopes is a hard task that need specific developments. In this paper we have seen that focal plane WFS has multiple features that make them good candidates for this task, especially for the cophasing of ELTs. The usual solution for cophasing ELTS (SH-WFS) uses interpolation algorithms and needs a dedicated hardware. We have proposed a new analytical algorithm that use the same device and can be used for a real-time cophasing. In addition, we showed that a larger number of segments can be cophased from 2 images by phase diversity. Indeed, with a pre-AO correction, a G0 star of magnitude 12 can be used to cophase an ELT with a precision of $4 \mathrm{~nm}$ at $\lambda=650 \mathrm{~nm}$. 
New analytical algorithms under development are expected to reduce the CPU load. In the beginning of 2010, we plan to demonstrate those algorithms on our bench NIRTA which is under integration at ONERA.

\section{References}

1. N. Yaitskova, F. Gonte, F. Derie, L. Noethe, I. Surdej, R. Karban, K. Dohlen, M. Langlois, S. Esposito, E. Pinna, M. Reyes, L. Montoya and D. Terrett, In Proceedings of the SPIE, Volume 6267, (2006), pp. 62672Z.

2. F. Gonte, N. Yaitskova, F. Derie, C. Araujo, R. Brast, B. Delabre, P. Dierickx, C. Dupuy, C. Frank, S. Guisard, R. Karban, L. Noethe, B. Sedghi, I. Surdej, R. Wilhelm, M. Reyes, S. Esposito and M. Langlois, In Proceedings of the SPIE, Volume 6267, (2006), pp. 626730 .

3. F. Gonte, In Adaptive optics for ELTs (2009).

4. G. Chanan, M. Troy, I. Crossfield, J. Nelson and T. Mast, In Proceedings of the SPIE, Volume 6267, (2006),pp. 62672V.

5. B. H. Dean, D. L. Aronstein, J. S. Smith, R. Shiri and D. S. Acton, In Proceedings of the SPIE, Volume 6265,(2006),pp. 626511.

6. G. Chanan, M. Troy, F. Dekens, S. Michaels, J. Nelson, T. Mast and D. Kirkman, In Appl. Opt., 37, (1998),pp. 140-155.

7. G. Chanan, C. Ohara and M. Troy, Phasing the Mirror Segments of the Keck Telescopes II: The Narrow-band Phasing Algorithm, In Appl. Opt., 39, (2000), pp. 4706-4714.

8. A. Schumacher, N. Devaney and L. Montoya, In Appl. Opt., 41, (2002), pp. 1297-1307 .

9. E. Delavaquerie and F. Cassaing, In Optics Letters, (to be submitted).

10. K. Houairi and Cassaing, In JOSA A, (Submitted).

11. A. Blanc, L. M. Mugnier and J. Idier, In JOSA A, 20, (2003), pp. 1035-1045.

12. S. Meimon, E. Delavaquerie, F. Cassaing, T. Fusco, L. M. Mugnier and V. Michau, In Proceedings of the SPIE, Volume 7012, (2008),pp. 701214.

13. F. Baron, I. Mocoeur, F. Cassaing and L. M. Mugnier, In JOSA A, 25, (2008), pp. 1000-1015.

14. I. Mocœur, L. Mugnier and F. Cassaing, In Optics Letters, (accepted), (2009).

15. F. Cassaing, B. Sorrente, L. Mugnier, G. Rousset, V. Michau, I. Mocœur and F. Baron, In Proceedings of the SPIE, Volume 6268, (2006),pp.62683A. 